$\mathrm{PM} / 98-16$

GDR-S-014

hep-ph/9807336

\title{
Inverting the Supersymmetric Standard Model Spectrum: from Physical to Lagrangian Ino Parameters
}

\author{
J.-L. Kneur I and G. Moultaka [2 \\ Physique Mathématique et Théorique, UMR No 5825-CNRS, \\ Université Montpellier II, F-34095 Montpellier Cedex 5, France.
}

\begin{abstract}
We examine the possibility of recovering the supersymmetric (and soft supersymmetry breaking) Lagrangian parameters as direct analytical expressions of appropriate physical masses, for the unconstrained (but CP and R-parity conserving) minimal supersymmetric standard model. We concentrate mainly on the algebraically nontrivial "inversion" for the ino parameters, and obtain, for given values of $\tan \beta$, simple analytical expressions for the $\mu, M_{1}$ and $M_{2}$ parameters in terms of three arbitrary input physical masses, namely either two chargino and one neutralino masses, or alternatively one chargino and two neutralino masses. We illustrate and discuss in detail the possible occurrence of ambiguities in this reconstruction. The dependence of the resulting ino Lagrangian parameters upon physical masses is illustrated, and some simple generic behaviour uncovered in this way. We finally briefly sketch generalizing such an inversion to the full set of MSSM Lagrangian parameters.
\end{abstract}

\footnotetext{
${ }^{1}$ kneur@lpm.univ-montp2.fr

2 moultaka@lpm.univ-montp2.fr
} 


\section{Introduction}

In the Minimal Supersymmetric extension of the Standard Model (MSSM) [1], 2], without additional theoretical assumptions, the necessary breakdown of supersymmetry leads to a large number of arbitrary parameters, consisting of all possible renormalizable soft supersymmetry breaking terms [3]. The latter arbitrariness simply reflects our present ignorance of the fundamental mechanism underlying the breakdown of supersymmetry. Although several theoretically appealing scenarios have been explored, with additional assumptions considerably reducing the arbitrariness of the soft SUSY-breaking sector (typically like in hidden sector supergravity models [4], or gauge-mediated susy breaking models [5]), alternative strategies may be useful to exploit the information from the present and future collider data. If SUSY is realized at low energy in some way, and some super-partners discovered, the next immediate task would be to reconstruct from the data the structure of the SUSY and soft-SUSY breaking Lagrangian [that is, to determine as precisely as possible how the experimentally measured parameters would translate as possible values (or bounds) on the MSSM Lagrangian parameters.]

The expressions of physical parameters (mass eigenvalues, mixing angles and physical couplings) as functions of the most general, unconstrained MSSM Lagrangian, are well known [1, []. Now, inverting those relations (i.e. expressing the Lagrangian parameters in terms of physical parameters) is non trivial in the unconstrained MSSM, due to the large number of parameters and possible mixing among them. This is especially true in the ino sector which involves a relatively complicated structure of the mixing terms, with e.g. a 4 x 4 mass matrix to "de-diagonalize" in the neutralino sector. To our knowledge, no systematic analytical inversion has been explored up to now.

The aim of the present paper is twofold:

- Investigate as much as possible analytically the reconstruction of the Lagrangian parameters from the physical masses, especially in the ino sector, identifying clearly the procedure and the related ambiguities when they occur;

- construct a numerical code based on algorithms using as much as possible the above mentioned results, and which can be readily used for a full numerical study of the Lagrangian parameters as a function of the physical masses.

For definiteness we will restrict ourselves throughout the paper to the (CP and Rparity conserving) unconstrained MSSM. Also to simplify the presentation we will consider two illustrative "scenarios" where either two chargino and one neutralino masses, or one chargino and two neutralino masses are input. The outcome is a well-defined algorithm providing, for given $\tan \beta$, the values of the $\mu, M_{1}$ and $M_{2}$ parameters in terms of three arbitrary input masses. Furthermore, one of our main results is the derivation, in terms of simple analytic expressions, of the full neutralino mass spectrum and one of

the Lagrangian parameters $\left(M_{1}\right)$, when the three other parameters $\left(M_{2}, \mu, \tan \beta\right)$ and one 
neutralino mass are given. This together with the analytical expressions of the chargino sector constitute the building blocks of our algorithms.

It should be stressed here that we are not aiming, in this paper, at a specific phenomenological reconstruction of the MSSM parameters from experimental observables. Clearly an algebraic approach cannot replace more systematic phenomenological studies of the reconstruction of the basic MSSM parameters. Various experimental strategies to extract the MSSM physical spectrum from present and future collider data have been considered, together with a detailed assessment on mass measurements, e.g., in ref. [7, 8]. On the other hand, a complete scanning of the unconstrained MSSM parameters would be in practice rather tedious and extremely time consuming. We believe that our study would be useful and rather flexible for such reconstructions: besides the fact of providing a fast numerical inversion routine, we systematically encode the possible ambiguities that can arise through the reconstruction from some of the physical masses. In the most optimistic case (that is, if knowing a sufficient number of physical input ino masses and with sufficient accuracy), this inversion allows for a precise reconstruction of the unconstrained MSSM ino sector Lagrangian. If a more limited experimental information of the ino spectrum were available, such as only mass bounds or mass differences typically, one could still derive corresponding constraints on the MSSM Lagrangian parameters as well.

Our input/output parameters are deliberately chosen so as to render the analytical inversion the most simple and transparent. As will be explained in details later on, our algorithms will be either fully analytical, in which case at most a readily solvable cubic equation is involved (in contrast with the generically quartic one in the ordinary diagonalization of the neutralino sector [9]), or needs some numerical iteration, in which case it relies essentially on a system of two algebraic equations, that are only quadratic (in contrast with a "brute force" inversion, which would involve a highly non-linear set of equations). It thus demonstrates the feasibility and relative simplicity of such a systematic inversion and more importantly we also exhibit the general trend and sensitivity of the Lagrangian parameters to the physical masses. Note, however, that we do not assume at this level any knowledge of the couplings of inos to higgses and gauge bosons. These would of course lead to extra information [10 which together with our results could allow an even more complete reconstruction and cross-checks for the ino sector.

The rest of the paper is organized as follows. In section 2, we briefly recall the main content of the MSSM Lagrangian, just to fix our definitions and conventions. In section 3, we present our main results for the analytic inversion of the ino parameters, and discuss uniqueness conditions. Some essentially technical material is given in two appendices. In section 4 we illustrate the dependence of the ino sector Lagrangian parameters, as functions of the relevant physical input masses. We also illustrate some resulting values of the ino Lagrangian parameters at a grand unification (GUT) scale. In section 5 we 
briefly outline, for completeness, similar inversion relations in the scalar fermion and Higgs sector of the MSSM. Finally section 6 presents the conclusions and an outlook.

\section{MSSM Lagrangian and spectrum: reminder}

The supersymmetric part of the unconstrained MSSM Lagrangian consists of the gauged kinetic terms for the $S U(3) \times S U(2) \times U(1)$ vector multiplets, involving the three gauge couplings $g_{3}, g_{2}$, and $g_{1}$, respectively, the gauge invariant kinetic term of matter fields, and the superpotential:

$$
W=\hat{\bar{u}} Y_{t} \hat{Q} \hat{H}_{u}-\hat{\bar{d}} Y_{b} \hat{Q} \hat{H}_{d}-\hat{\bar{e}} Y_{\tau} \hat{L} \hat{H}_{d}+\mu \hat{H}_{u} \hat{H}_{d}
$$

In eq. (2.1), the hats denote superfields, $Y_{i}$ are the Yukawa couplings, and $\hat{H}_{u} \hat{H}_{d} \equiv$ $\epsilon_{i j} \hat{H}_{u}^{i} \hat{H}_{d}^{j}$, (with $\epsilon_{12}=1$ ) fixes our sign convention for $\mu$. In (2.1) and in the following we will in fact neglect all flavor non-diagonal terms. We also suppress any flavor, color, etc indices. Moreover, we will restrict here to the case where all parameters are assumed to be real.

The soft SUSY-breaking terms involve -the trilinear coupling terms:

$$
\mathcal{L}_{\text {trilinear }}=-\left(\tilde{u} A_{t} Y_{t} H_{u} \tilde{Q}+\tilde{d} A_{b} Y_{b} H_{d} \tilde{Q}+\tilde{e} A_{\tau} Y_{\tau} H_{d} \tilde{L}+\text { h.c. }\right)
$$

involving the $A_{i}$ parameters (which have a mass dimension in this convention); -a contribution to the sfermion mass terms:

$$
\begin{array}{r}
\mathcal{L}_{\text {sfermion }}=-M_{Q}^{2}\left(\tilde{t}_{L}^{\star} \tilde{t}_{L}+\tilde{b}_{L}^{\star} \tilde{b}_{L}\right)-M_{t_{R}}^{2} \tilde{t}_{R}^{\star} \tilde{t}_{R}-M_{b_{R}}^{2} \tilde{b}_{R}^{\star} \tilde{b}_{R} \\
-M_{L}^{2}\left(\tilde{\tau}_{L}^{\star} \tilde{\tau}_{L}+\tilde{\nu}_{L}^{\star} \tilde{\nu}_{L}\right)-M_{\tau_{R}}^{2} \tilde{\tau}_{R}^{\star} \tilde{\tau}_{R}
\end{array}
$$

and the gaugino mass terms:

$$
\mathcal{L}_{\text {gaugino }}=-\frac{M_{1}}{2} \tilde{B} \tilde{B}-\frac{M_{2}}{2} \tilde{W}^{i} \tilde{W}_{i}-\frac{M_{3}}{2} \tilde{G}^{a} \tilde{G}_{a}
$$

which fixes our sign conventions. There are also supersymmetric contributions to the ino masses, of which we write here for illustration only the ones contributing to neutralinos (after electroweak symmetry breaking):

$$
\begin{aligned}
\mathcal{L}_{\text {neutralino }}= & m_{Z} c_{w} \sin \beta \tilde{W}_{3} \tilde{H}_{u}-m_{Z} c_{w} \cos \beta \tilde{W}_{3} \tilde{H}_{d} \\
& +m_{Z} s_{w} \cos \beta \tilde{B} \tilde{H}_{d}-m_{Z} s_{w} \sin \beta \tilde{B} \tilde{H}_{u}+\mu \tilde{H}_{u} \tilde{H}_{d}
\end{aligned}
$$

with $s_{w} \equiv \sin \theta_{W}, c_{w} \equiv \cos \theta_{W}$, and $\tan \beta \equiv v_{u} / v_{d}$ the ratio of the two Higgs vacuum expectation values, $v_{u, d}=\left\langle H_{u, d}\right\rangle$. It is now easy to see from Eqs.(2.4, 2.5) that only the 
relative phases of say $\left(M_{2}, M_{1}\right)$ and $\left(M_{2}, \mu\right)$ are physically relevant. Indeed any phase change of $M_{2}$ in Eq.(2.4) can always be absorbed by a phase change of the $\tilde{W}$ field. The latter however fixes uniquely the phase change of $\tilde{H}_{d}, \tilde{H}_{u}$ and $\tilde{B}$ in Eq.(2.5) in such a way that the phases of the combinations $M_{2} / M_{1}$ and $\mu M_{2}^{2}$ remain unchanged. The discussion is of course reminiscent of the one carried out for instance in [2]. In the present study, as already mentioned, we restrict ourselves to real-valued parameters, but do not necessarily assume universality of gaugino masses. We can thus choose, without loss of generality, $M_{2}$ to be always positive and $M_{1}$ and $\mu$ to have arbitrary signs.

Finally, the Higgs potential is built from soft SUSY breaking and F-term contributions to the Higgs scalar mass terms plus quadrilinear D-terms, and reads, before $S U(2) \times U(1)$ breaking:

$$
\begin{array}{r}
V_{\text {Higgs }}=\left(m_{H_{d}}^{2}+\mu^{2}\right)\left|H_{d}\right|^{2}+\left(m_{H_{u}}^{2}+\mu^{2}\right)\left|H_{u}\right|^{2}+\left(B \mu H_{u} H_{d}+\text { h.c. }\right) \\
+\frac{g_{1}^{2}+g_{2}^{2}}{8}\left(\left|H_{d}\right|^{2}-\left|H_{u}\right|^{2}\right)^{2}+\frac{g_{2}^{2}}{2}\left|H_{d}^{\star i} H_{u, i}\right|^{2}+V_{\text {eff }}^{1-\text { loop }}
\end{array}
$$

which also fixes a sign convention for the $B$ parameter, and where $V_{\text {eff }}^{1-l o o p}$ is the one-loop contribution to the effective potential [11, 12, 13, 14]. The corresponding Higgs masses and mixing angles (after electroweak symmetry breaking) are given in Appendix 1.

\section{Inverting the ino MSSM spectrum}

Let us first sketch our general procedure to reconstruct the ino sector parameters. As already mentioned in the introduction, we have to fix a specific choice of input/output parameters, that we do as follows. We first assume that $\tan \beta$ is an input parameter at this stage, i.e. that it has been extracted from elsewhere prior to ino reconstruction, or simply fixed arbitrarily. (Obviously, once a reconstruction algorithm is defined for fixed $\tan \beta$, one may always study the sensitivity to this parameter). Then, we consider two basic scenarios, $S_{1}$ and $S_{2}$, and the corresponding algorithms.

$S_{1}$ : the input are two chargino masses, $M_{\chi_{1}^{+}}, M_{\chi_{2}^{+}}$, and one (but any) of the neutralino masses, $\pm M_{N_{i}}$.

$S_{2}$ : the input are a single chargino mass, $M_{\chi_{1}^{+}}$, and two neutralino masses, say $\pm M_{N_{2}}$, $\pm M_{N_{3}}$.

It is important to note here that we will adopt the formulation in which the eigenvalues of the chargino mass matrix are by construction always positive, while those of the neutralino mass matrix can have either signs. This means that in the inversion process one should consider both $\pm M_{N_{i}}$ as possible inputs [a feature consistently taken into account in the formulae we derive.] 
For each of the above scenarios the aim will be to identify a corresponding algorithm which allows the determination of the output values of $\mu, M_{2}, M_{1}$, as well as the remaining three neutralino masses (resp. two neutralinos and one chargino masses) in the $S_{1}$ (resp. $\left.S_{2}\right)$ case.

First, as we will show in detail below and in Appendix $\mathrm{B}$, scenario $S_{1}$ allows a fully analytical algorithm to handle all the features of the inversion procedure. Algorithm $S_{1}$ will therefore be our starting point for the whole study 1. We stress, however, that it does not necessarily imply a strong prejudice on the "chronology" of discovery of the inos since, as we shall explain, it may also be used as a basic building block for a probably more phenomenologically relevant situation, in scenario $S_{2}$ below. [Furthermore, we will see that $S_{1}$ can be naturally separated into two independent steps corresponding to chargino and neutralino sectors. It can thus also be readily used in a more general context than the one of the present paper].

To scenario $S_{2}$ we associate the following algorithm: first assuming temporarily that e.g. $\mu$ is an input parameter, together with two neutralino masses, say $M_{N_{2}}$ and $M_{N_{3}}$, then a simple quadratic system gives $M_{2}, M_{1}$ as function of the latter input, as well as all other ino masses. Now, the key observation is that it is relatively simple to merge the solutions of the latter system with the previous algorithm $S_{1}$, to obtain consistently $\mu, M_{2}, M_{1}$ from a single chargino mass, $M_{\chi_{1}^{+}}$, and two neutralino masses, say $M_{N_{2}}, M_{N_{3}}$ input. More precisely, choosing an adequate "initial guess" value for e.g. $M_{2}$ one can simply use $S_{1}$ (actually only a part of it) to determine $\mu$ in terms of $\left(M_{\chi_{1}^{+}}, M_{2}\right)$, followed by the above mentioned $M_{1}, M_{2}$ solutions, iterating with respect to $M_{2}$ until a convergent set of values is obtained 2 . While $S_{1}$ was fully analytical, the price to pay is that, accordingly, $S_{2}$ has to be partly numerical through the iterative procedure. In most cases, this combined algorithm turns out to converge rapidly, after 2 to 3 iterations for an accuracy that is sufficient for all practical purposes.

This particular decomposition, with this choice of input/output, is the one giving the most algebraically tractable inversion in the ino sector. In fact, while $S_{1}$ alone is probably not very relevant physically (since for a rather generic choice of the Lagrangian parameters, it looks more likely to have two neutralinos and one chargino in the lightest part of the ino spectrum [7, 2, 14]), precisely this situation is tractable from the combined algorithm of scenario $S_{2}$, as explained above. However, it is rather instructive to study algorithm $S_{1}$ separately in some detail, as it exhibits important properties of the inversion in a relatively simple manner. [Of course it is also possible to use any part of our particular

\footnotetext{
${ }^{1}$ Except when ambiguous, we call from now on the scenario and its associated algorithm with the same name.

${ }^{2}$ There are always (complex) solutions since, as easily checked, the explicit non-linear system equivalent to $S_{2}$ is a (high degree) polynomial. Non convergent domains thus simply correspond to the impossibility to match our basic assumption of real $\mu, M_{1}, M_{2}$.
} 
procedures to simply scan over some values, if not known, of some of the ino masses, as will be illustrated in the plots in section 4].

\subsection{Basic algorithm $S_{1}: M_{\chi_{1}^{+}}, M_{\chi_{2}^{+}}, M_{N_{2}}$ input}

Assuming $\tan \beta$ and the two chargino masses given, one easily obtains from the expressions of the chargino mass eigenvalues (see eq (A.2) in Appendix A $)$ :

$$
\mu^{2}=\frac{1}{2}\left(M_{\chi_{1}^{+}}^{2}+M_{\chi_{2}^{+}}^{2}-2 m_{W}^{2} \pm\left[\left(M_{\chi_{1}^{+}}^{2}+M_{\chi_{2}^{+}}^{2}-2 m_{W}^{2}\right)^{2}-4\left(m_{W}^{2} \sin 2 \beta \pm M_{\chi_{1}^{+}} M_{\chi_{2}^{+}}\right)^{2}\right]^{1 / 2}\right)
$$

and

$$
M_{2}=\left[M_{\chi_{1}^{+}}^{2}+M_{\chi_{2}^{+}}^{2}-2 m_{W}^{2}-\mu^{2}\right]^{1 / 2}
$$

In eqs. (3.1) and (3.2) the \pm ambiguity in front of the square root results from the invariance of the physical masses (see eq. (A.2) of appendix A) under the substitution $|\mu| \leftrightarrow M_{2}$ (we stress again our sign convention, $M_{2}>0$ in (3.2)). In other words, from the two chargino masses input only, there is clearly no way of assessing the amount of gaugino or higgsinos components of each of them. To achieve this, one would require the knowledge of mixing angles (i.e. couplings) [10 or alternatively of two neutralino masses, as will be discussed below in scenario $S_{2}$. In what follows, we thus arbitrarily choose to illustrate only the case $|\mu| \leq M_{2}$ (corresponding to the minus sign choice in front of the square root of (3.1)), i.e. the case where the lightest chargino has a stronger higgsino like component. We thus refer to this situation as "higgsino-like". The resulting output of algorithm $S_{1}$ corresponding to the opposite "gaugino-like" situation, $|\mu| \geq M_{2}$, is therefore trivially obtained by interchanging the values of $|\mu|$ and $M_{2}$ (together with the correct sign of $\mu$ assignment, see e.g. captions of fig. A). In addition, since $M_{2}>0$, $M_{1}$ will have arbitrary sign (see previous section 2). [Had we chosen to let the sign of $M_{2}$ rather than $M_{1}$ undetermined at this stage, our algorithm would have been slightly more complicated].

Concerning now the sign ambiguity on $\mu$, it is in fact fixed from another relation from eqs. (A.2) (implicitly used in eq. (3.1)):

$$
M_{2} \mu=m_{W}^{2} \sin 2 \beta \pm M_{\chi_{1}^{+}} M_{\chi_{2}^{+}}
$$

for each respective choice of the \pm ambiguity. The latter \pm , also appearing inside the square root in (3.1), corresponds to a true ambiguity, i.e. whenever the expression under the square root of eq. (3.1) is positive for both sign choice, there are two distinct solutions for $\left(\mu, M_{2}\right)$. Obviously, the occurrence of this discrete twofold ambiguity depends crucially on the values of $M_{\chi_{1}^{+}}, M_{\chi_{2}^{+}}$and $\tan \beta$, and deserves a more careful examination to which we now turn.

We illustrate in table 1 and fig.1 the different domains where one obtains either no solutions (for $\mu$ real), a unique solution, or the discrete twofold ambiguity, depending 
on the input values $M_{\chi_{1}^{+}}, M_{\chi_{2}^{+}}$and $\tan \beta$. What is relevant here are the chargino mass difference $\Delta_{\chi^{+}} \equiv\left|M_{\chi_{2}^{+}}-M_{\chi_{1}^{+}}\right|$and sum $\Sigma_{\chi_{+}} \equiv M_{\chi_{1}^{+}}+M_{\chi_{2}^{+}}$, as well as the quantity

$$
X_{\epsilon_{2}}^{\epsilon_{1}}=-\frac{\epsilon_{1} 2 M_{\chi_{1}^{+}} M_{\chi_{2}^{+}}+\epsilon_{2}\left(M_{\chi_{1}^{+}}^{2}+M_{\chi_{2}^{+}}^{2}-2 M_{W}^{2}\right)}{2 M_{W}^{2}},
$$

where $\epsilon_{1}, \epsilon_{2}= \pm 1$. For instance, the last two columns of table 1 summarize respectively the occurrence of the twofold ambiguity or the existence of (real) solutions at all. In some zones this is fully controlled by the values of $\tan \beta$ which we expressed in the form of necessary and sufficient constraints on $\sin 2 \beta$. This is systematically taken into account in our algorithm, which gives in the relevant case the two possible solutions for $\mu$. One then needs an extra information to eventually eliminate one of the solutions. Note finally that in fig.11 we assumed for simplicity that $M_{\chi_{2}^{+}}>M_{\chi_{1}^{+}}$which is obviously just a matter of convention. [In any case, the opposite situation corresponds simply to a symmetry around the bisectrix line.]

\begin{tabular}{|c|c|c|l|l|l|}
\hline \hline$\Delta_{\chi^{+}} / \sqrt{2} M_{W}$ & $\Delta_{\chi^{+}} / 2 M_{W}$ & $\Sigma_{\chi_{+}} / 2 M_{W}$ & $\begin{array}{l}X_{+}^{+}<0 ; X_{+}^{-}<1 \\
\text { in all cases }\end{array}$ & $\begin{array}{l}\text { twofold } \\
\text { ambiguity }\end{array}$ & $\begin{array}{l}\text { constraint } \\
\text { on } \beta\end{array}$ \\
\hline $\begin{array}{c}\text { zone (I) } \\
<1\end{array}$ & $<1$ & $<1$ & $\begin{array}{l}0<X_{ \pm}^{-}<1 \\
X_{-}^{+}<0\end{array}$ & No & $X_{+}^{-}<\sin 2 \beta<X_{-}^{-}$ \\
\hline $\begin{array}{c}\text { zone (II) } \\
>1\end{array}$ & $<1$ & $<1$ & $\begin{array}{l}0<X_{+}^{-}<1<X_{-}^{-} \\
X_{-}^{+}<0\end{array}$ & No & sin $2 \beta>X_{+}^{-}$ \\
\hline$>1$ & $<1$ & $>1$ & $\begin{array}{l}X_{+}^{-}<0 ; 1<X_{-}^{-} \\
0<X_{-}^{+}<1\end{array}$ & $\begin{array}{l}\text { Yes when } \\
\sin 2 \beta<X_{-}^{+}\end{array}$ & No \\
\hline $\begin{array}{c}\text { zone (III) } \\
>1\end{array}$ & $>1$ & $>1$ & $\begin{array}{l}X_{+}^{-}<0 ; 1<X_{-}^{-} \\
X_{-}^{+}>1\end{array}$ & Yes & No \\
\hline zone (IV) & & $>1$ & &
\end{tabular}

Table 1: Inequalities characterizing the different domains of solutions $\mu, M_{2}$ for arbitrary $M_{\chi_{1}^{+}}, M_{\chi_{2}^{+}}$input. $\Delta_{\chi^{+}} \equiv\left|M_{\chi_{2}^{+}}-M_{\chi_{1}^{+}}\right| ; \Sigma_{\chi^{+}} \equiv M_{\chi_{1}^{+}}+M_{\chi_{2}^{+}} . X_{ \pm}^{ \pm}$is defined in the text.

\subsection{Neutralino mass inversion}

Let us now turn to the de-diagonalization of the neutralino sector. The question we want to answer analytically here, is how to determine $M_{1}$ and three neutralino masses, when $M_{2}, \mu, \tan \beta$ and a fourth neutralino mass are given. We will only outline the procedure leaving some technical details to the appendix. As we shall see, the discussion in this section is quite general and serves indifferently as a basis for the more specific algorithm $S_{1}$ or $S_{2}$. 


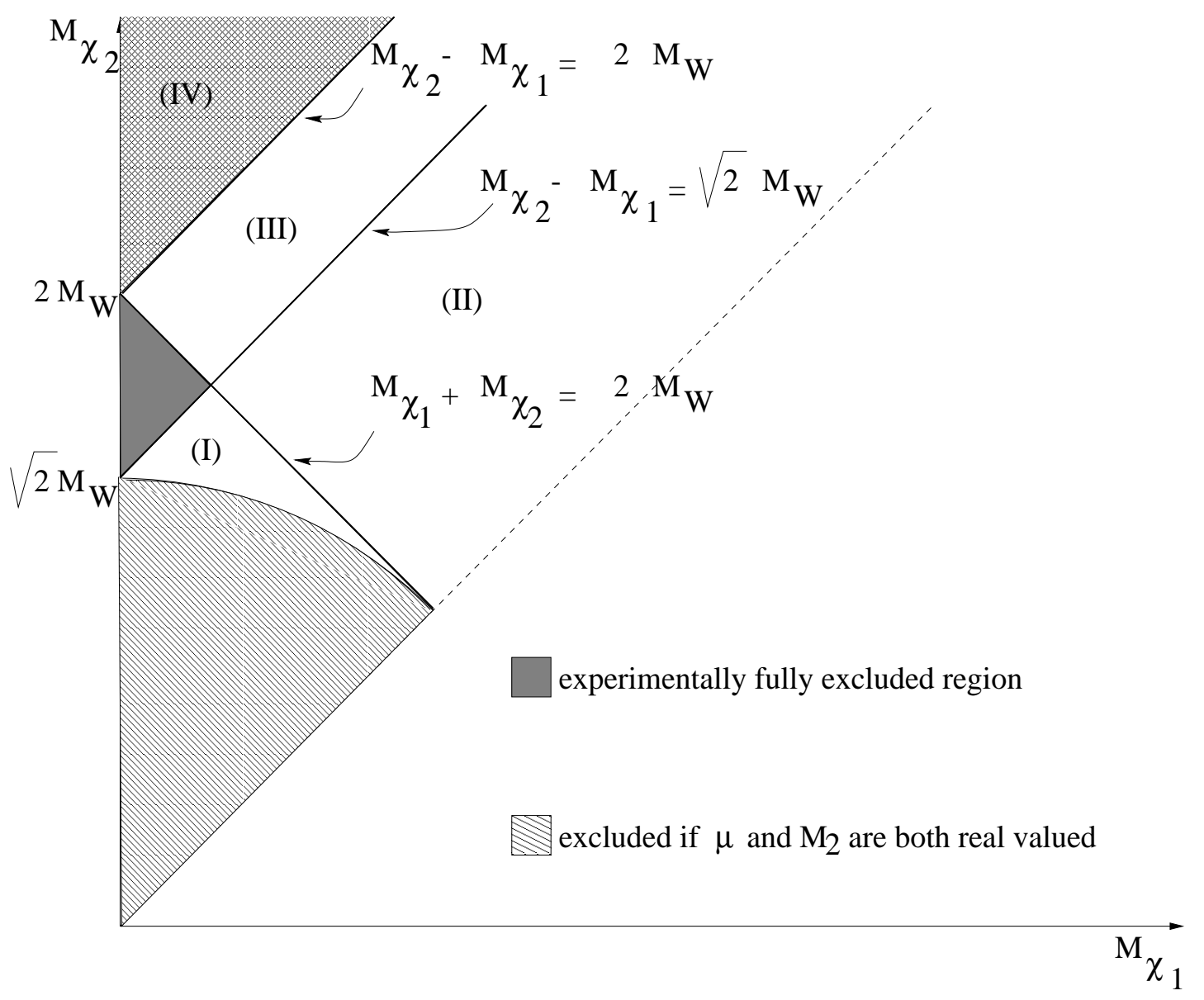

Figure 1: Domains of solutions for $\mu, M_{2}$ as functions of $M_{\chi_{1}^{+}}, M_{\chi_{2}^{+}}$and $\tan \beta$, where we assumed without loss of generality $M_{\chi_{2}^{+}}>M_{\chi_{1}^{+}}$. The detailed definitions and properties of the different domains (I)-(IV) are explained in Table 1.

Since we restrict ourselves to the case where $M_{1}, M_{2}$ and $\mu$ are all real-valued, the neutralino mass matrix

$$
M=\left(\begin{array}{cccc}
M_{1} & 0 & -m_{Z} s_{W} \cos \beta & m_{Z} s_{W} \sin \beta \\
0 & M_{2} & m_{Z} c_{W} \cos \beta & -m_{Z} c_{W} \sin \beta \\
-m_{Z} s_{W} \cos \beta & m_{Z} c_{W} \cos \beta & 0 & -\mu \\
m_{Z} s_{W} \sin \beta & -m_{Z} c_{W} \sin \beta & -\mu & 0
\end{array}\right)
$$

is symmetric and can be diagonalized through a similarity transformation, i.e.

$$
P M P^{-1}=M_{\text {diagonal }}
$$

Analytical results for this diagonalization are well-known 9]. However, a straightforward inversion of the expressions of the mass eigenvalues using these results is far from obvious. 
A more manageable alternative is to start from the four quantities

$$
\begin{aligned}
& \operatorname{Tr} M \\
& \frac{(\operatorname{Tr} M)^{2}}{2}-\frac{\operatorname{Tr}\left(M^{2}\right)}{2} \\
& \frac{(\operatorname{Tr} M)^{3}}{6}-\frac{\operatorname{Tr} M \operatorname{Tr}\left(M^{2}\right)}{2}+\frac{\operatorname{Tr}\left(M^{3}\right)}{3} \\
& \operatorname{Det} M
\end{aligned}
$$

which are invariant under any similarity transformation. These quantities allow one to relate the four mass eigenvalues to the initial parameters in (3.5) as follows:

$$
\begin{gathered}
\tilde{M}_{N_{1}}+\tilde{M}_{N_{2}}+\tilde{M}_{N_{3}}+\tilde{M}_{N_{4}}=M_{1}+M_{2} \\
\tilde{M}_{N_{1}} \tilde{M}_{N_{2}}+\tilde{M}_{N_{2}} \tilde{M}_{N_{3}}+\tilde{M}_{N_{3}} \tilde{M}_{N_{4}}+\tilde{M}_{N_{4}} \tilde{M}_{N_{1}}=M_{1} M_{2}-\mu^{2}-M_{Z}^{2} \\
\tilde{M}_{N_{1}} \tilde{M}_{N_{2}} \tilde{M}_{N_{3}}+\tilde{M}_{N_{2}} \tilde{M}_{N_{3}} \tilde{M}_{N_{4}}+\tilde{M}_{N_{3}} \tilde{M}_{N_{4}} \tilde{M}_{N_{1}}+\tilde{M}_{N_{4}} \tilde{M}_{N_{1}} \tilde{M}_{N_{2}} \\
=\mu M_{Z}^{2} \sin 2 \beta-\left(\mu^{2}+c_{w}^{2} M_{Z}^{2}\right) M_{1}-\left(\mu^{2}+s_{w}^{2} M_{Z}^{2}\right) M_{2} \\
\tilde{M}_{N_{1}} \tilde{M}_{N_{2}} \tilde{M}_{N_{3}} \tilde{M}_{N_{4}}=\mu M_{Z}^{2}\left(c_{w}^{2} M_{1}+s_{w}^{2} M_{2}\right) \sin 2 \beta-\mu^{2} M_{1} M_{2}
\end{gathered}
$$

Here $\tilde{M}_{N_{i}}$ denote the eigenvalues of the mass matrix Eq.(3.5), to be distinguished from the physical neutralino masses $M_{N_{i}}$ given by $\tilde{M}_{N_{i}} \equiv \epsilon_{i} M_{N_{i}}$, where $\epsilon_{i}= \pm$ is undetermined at this level.

We emphasize that Eqs. 3.11 3.14) give the complete information on the relationship between the four neutralino mass eigenvalues and the original parameters, $\mu, M_{1}, M_{2}$ and $\tan \beta$. Furthermore these equations are a good starting point for our purpose, as they do not favour a priori any particular set of parameters. Thus, the system may be solved in many different ways, depending on the choice of input/output one is interested in.

According to the algorithm $S_{1}$, described in the previous section, we can assume that $\mu, M_{2}$ (and $\left.\tan \beta\right)$ are determined at this stage, and extract $M_{1}$ and the three physical masses $M_{N_{1}}, M_{N_{3}}, M_{N_{4}}$ as functions of one mass, say $M_{N_{2}}$.

Accordingly, in this $S_{1}$ scenario, one finds for $M_{1}$

\footnotetext{
${ }^{3}$ Actually what we call $M_{N_{2}}$ plays the role of any neutralino mass to be given as input. There will be a relabeling of neutralino states depending on the values of other parameters, and one can check afterwards whether the input mass was the lightest, heaviest or intermediate one.
} 


$$
M_{1}=-\frac{P_{2 i}^{2}+P_{2 i}\left(\mu^{2}+M_{Z}^{2}+M_{2} S_{2 i}-S_{2 i}^{2}\right)+\mu M_{Z}^{2} M_{2} s_{w}^{2} \sin 2 \beta}{P_{2 i}\left(S_{2 i}-M_{2}\right)+\mu\left(c_{w}^{2} M_{Z}^{2} \sin 2 \beta-\mu M_{2}\right)}
$$

where

$$
\begin{gathered}
S_{2 i} \equiv \tilde{M}_{N_{2}}+\tilde{M}_{N_{i}} \\
P_{2 i} \equiv \tilde{M}_{N_{2}} \tilde{M}_{N_{i}}
\end{gathered}
$$

and $\tilde{M}_{N_{i}}$ is any of the three remaining neutralino mass parameters.

The latter take the following form,

$$
\begin{gathered}
\tilde{M}_{N_{1}}=-\frac{1}{3 a_{1}}\left(a_{2}-2 \sqrt{-A} \cos (\operatorname{Arg}[B])\right) \\
\tilde{M}_{N_{3}}=-\frac{1}{3 a_{1}}\left(a_{2}+2 \sqrt{-A} \cos \left(\operatorname{Arg}[B]-\frac{\pi}{3}\right)\right) \\
\tilde{M}_{N_{4}}=-\frac{1}{3 a_{1}}\left(a_{2}+2 \sqrt{-A} \cos \left(\operatorname{Arg}[B]+\frac{\pi}{3}\right)\right)
\end{gathered}
$$

where A, B, $a_{1}$ and $a_{2}$ are given in Appendix B, Eqs.(B.7, B.11, B.12), and $\operatorname{Arg}[B]$ denotes the phase of the generically complex valued quantity $B$. Note that $M_{1}, \tilde{M}_{N_{1}}, \tilde{M}_{N_{3}}$ and $\tilde{M}_{N_{4}}$ are all real-valued since we chose $\mu$ and $M_{2}$ to be real. Note also that Eq.(3.15) should yield the same value when $\tilde{M}_{N_{i}}$ is substituted by any of the three neutralino masses given by Eqs. (3.16 - 3.18). This allows a non-trivial consistency check. For a more detailed discussion of the derivation of Eqs.(3.15 - 3.18) and related material the reader is referred to Appendix B.

\subsection{Combined algorithm scenario $S_{2}: M_{\chi_{1}^{+}}, M_{N_{2}}, M_{N_{3}}$ input}

Alternatively, when considering now scenario $S_{2}$ as defined previously (i.e. the inputs are $\mu, M_{N_{2}}, M_{N_{3}}$ ), an even simpler solution occurs for $M_{2}$ and $M_{1}$ from eqs (3.11)-(3.14). In fact, the necessary and sufficient conditions for the existence of solutions to eq. (3.11)(3.14) give the consistency conditions eqs (B.4), (B.5) (see Appendix B), from which one immediately obtains, for given $\mu$, simple quadratic equations for $M_{2}, M_{1}$, that we omit to write here. The price to pay, as already mentioned previously, is that without further model-dependent assumption, this combined algorithm for scenario $S_{2}$ has to rely on a numerical (iterative) consistency check, and some non-trivial procedure has to be performed in order not to miss all possible solutions f. The upshot is that up to at most four distinct solutions for $\left(\mu, M_{1}, M_{2}\right)$ can occur for given $M_{\chi_{1}^{+}}, M_{N_{2}}, M_{N_{3}}$ input, once

\footnotetext{
${ }^{4}$ Indeed, solving e.g. eq. (3.1) for $\mu$ as a function of $M_{\chi_{1}^{+}}, M_{2}$, and bravely injecting the solution in eqs. (B.4)-(B.5) gives a highly non-linear equation for, e.g., $M_{2}$. Most of the solutions are in fact spurious, and our iteration algorithm, based on two equations which are only quadratic, copes with these redundancies in a simpler way.
} 
all consistency constraints (including our sign convention choice $M_{2}>0$ ) are taken into account. Unfortunately, in contrast with the twofold ambiguities of algorithm $S_{1}$ alone, it is not easy to work out analytically specific domains of the input parameter space corresponding to a definite number of distinct solutions, and one has to rely on the numerics.

Let us summarize this section:

-in scenario $S_{1}$, we start from $M_{\chi_{1}^{+}}, M_{\chi_{2}^{+}}, M_{N_{2}}$ to determine first $\mu$ and $M_{2}$ (up to the possible twofold ambiguity), and then $M_{1}$ from eq. (3.15), given $\tan \beta$. The solution for $M_{1}$, (3.15), is indeed unique for fixed $M_{2}, \mu$ and one neutralino mass values. This part of the algorithm may thus be used in a more general context (than $S_{1}$ ), where $\mu, M_{2}$ and $\tan \beta$ would have been extracted in a way or the other. Within scenario $S_{1}$ alone, there are in principle two possible $M_{1}$ values for any two chargino and one neutralino mass input, since the chargino mass input does not distinguish the gaugino-like from the higgsino-like situation, and (3.15) is not symmetrical under the interchange $|\mu| \leftrightarrow M_{2}$. -Alternatively, in scenario $S_{2}$ one can obtain $\mu, M_{2}, M_{1}$ consistently by iteration from $M_{\chi_{1}^{+}}, M_{N_{2}}$, and $M_{N_{3}}$. In practice, this combined algorithm converges very rapidly, but a relatively large number of distinct solutions (up to four) can a priori occur in some domains of the relevant input parameter space $\left(\mu, M_{1}, M_{2}\right)$. [Note that these ambiguities are different from the previous Higgsino-like $\leftrightarrow$ gaugino-like ambiguity of scenario $S_{1}$, the latter being precisely removed from the knowledge of a second neutralino mass].

\section{Numerical illustrations of the ino inversion}

We shall illustrate here with some representative plots the results of the inversion in the ino sector, according to the algorithms explained in section 3, together with a few remarks and comments. Since our choice of input masses is rather arbitrary and may not directly correspond to the most interesting experimental situation, our comments are accordingly essentially qualitative. We will see, nevertheless, that a number of general and interesting properties of the inversion can directly be seen here, irrespective of the precise values of the other parameters that have to be fixed, like $\tan \beta$ typically.

\subsection{Scenario $S_{1}$ : two charginos plus one neutralino input}

We first illustrate the basic algorithm $S_{1}$, namely the reconstruction of $\mu, M_{2}$ (and $M_{1}$ ) from $M_{\chi_{1}^{+}}, M_{\chi_{2}^{+}}$(and $\tan \beta$ and $M_{N_{2}}$ ) in fig. 2. As already mentioned, we illustrate only the higgsino-like situation $|\mu| \leq M_{2}$ since the gaugino-like illustration is trivially obtained from it (see captions of fig. 2).

Since it is phenomenologically more likely [0] that the first inos discovered (if any) will be two neutralinos and only one chargino (the second chargino being of heavier mass), we fixed in scenario $S_{1}$ only one chargino mass, say $M_{\chi_{1}^{+}}$, while varying the other one, $M_{\chi_{2}^{+}}$, in a large range to illustrate as much as possible the dependence on the physical input 
(see figure captions). The shape of the various plots in fig. 2 is quite generic. First, it exhibits three distinct zones as regards the existence, uniqueness, or possible ambiguities on $\mu, M_{2}, M_{1}$ (see also the discussion and table 1 in section 3 ):

i) the grey shaded region, where there are no solutions for real $\mu$, corresponds to zone (I) of table 1 , with the corresponding constraint on $\sin 2 \beta$ not fulfilled f If one takes a smaller or larger $M_{\chi_{1}^{+}}$, this region around $M_{\chi_{1}^{+}}$will be simply displaced accordingly.

ii) in the left and right boarder zones are the regions of twofold ambiguities on $\mu, M_{2}$, as indicated. Note therefore that one of the two solutions has a discontinuity at the boarder between the single and twofold solutions region. Without additional information (or particular model assumption) one cannot a priori reject any of the two solutions.

iii) Finally the two bands in between zones i) and ii) correspond to the region where eqs. (3.1, 3.2) give a unique solution for $\mu$ and $M_{2}$. It is interesting to note that those bands are narrower when $\tan \beta$ is increasing (in fig. $2 \tan \beta=2$ ), irrespective of the $M_{\chi_{1}^{+}}$values, becoming e.g. only a few $\mathrm{GeV}$ wide for $\tan \beta>35$.

Moreover, as a general behaviour, the values of $\mu$ and $M_{2}$ are rather insensitive to $\tan \beta$ (apart obviously from the discontinuous change occurring for one of the solution at the boarder between zones ii) and iii)). One can also remark from fig. 2 the relatively simple shape of $\mu$ and $M_{2}$ as function of $M_{\chi_{2}^{+}}$, with an almost constant or linear dependence, apart from some narrow regions. This is easily understood, since from eqs. (3.1), (3.2) one obtains $\left(\mu, M_{2}\right) \simeq\left(M_{\chi_{2}^{+}}, M_{\chi_{1}^{+}}\right)$, (resp. $\left(M_{\chi_{1}^{+}}, M_{\chi_{2}^{+}}\right)$) for $M_{\chi_{2}^{+}}<<M_{\chi_{1}^{+}}$(resp. $M_{\chi_{2}^{+}}>M_{\chi_{1}^{+}}$.

In fig. 2 we also plot $M_{1}$ for the corresponding values of $\mu$ and $M_{2}$, and for fixed $M_{N_{2}}$. The twofold valuedness of $M_{1}$ there is entirely due to the twofold ambiguity of $\mu$ and $M_{2}$. In contrast, we do not illustrate here the other determination of $M_{1}$ resulting from the $|\mu| \leftrightarrow M_{2}$ interchange: although the resulting $M_{1}$ may be in general quite different, this interchange will have very little effects as a function of $M_{\chi_{2}^{+}}$. First of all, the trivial, almost constant behaviour of $M_{1}$ in this plot (except for small $M_{\chi_{2}^{+}} \lesssim 100$ $\mathrm{GeV}$ ) is relatively simple to understand from eq. (3.15). Indeed, $M_{2}$ is large for large $M_{\chi_{2}^{+}}$ (since we assume $|\mu|<M_{2}$ ). In this limit and for fixed $M_{\chi_{1}^{+}}, M_{N_{2}}$ (and $\tan \beta$ ) one finds from eqs.(B.7-B.10) that the remaining three neutralinos behave like $M_{2}$. One then easily determines the (constant) behaviour of $M_{1}$ from eq.(3.15) in the large $M_{2}$ limit, namely $M_{1} \simeq\left[M_{N_{2}}\left(M_{N_{2}}^{2}-\mu^{2}-m_{Z}^{2}\right)-s_{w}^{2} \mu m_{Z}^{2} \sin 2 \beta\right] /\left(M_{N_{2}}^{2}-\mu^{2}\right)$

\footnotetext{
${ }^{5}$ Of course, more generally one could be interested in complex $\mu$ solution. The results of section 3.2 and appendix B, not directly applicable in this case, would then need a generalization which is beyond the scope of the present paper.
} 
In summary, the information from the plots in fig. 2 is rather interesting: apart from some small regions, the dependence of $\mu, M_{2}$ (and even $M_{1}$ to some extent) upon $\left|M_{\chi_{1}^{+}}-M_{\chi_{2}^{+}}\right|$is very simple for a wide range of the latter mass difference. In other words, once one chargino mass will be known with some accuracy (together with $\tan \beta$ ), fig. 2 indicates that, in principle, the possible values of $\mu, M_{2}$, and $M_{1}$ are strongly correlated. Moreover, although in fig 2 we have only varied $M_{\chi_{2}^{+}}$the behaviour is quite generic and does not change much, qualitatively, when the other fixed inputs $\left(M_{N_{2}}\right.$ and $\left.\tan \beta\right)$ are varied.

Finally, as a last illustration of scenario $S_{1}$, we plot in fig. 3 the resulting values of $M_{1}$ and the three other neutralino masses, $M_{N_{1}}, M_{N_{3}}, M_{N_{4}}$, determined from eqs. (3.16)(3.18). Note that the plots in fig. 3 are now functions of $M_{N_{2}}$, and with different $M_{\chi_{1}^{+}}$, $M_{\chi_{2}^{+}}$inputs than for fig. 2. The singularities of $M_{1}$ for specific values of $M_{N_{2}}$, correspond simply to the denominator of eq.(3.15) vanishing (and also correspond to the exchange among neutralino masses, as illustrated on the plots). Apart from relatively small regions around the singularities, the almost linear dependence of $M_{1}$ as a function of $M_{N_{2}}$ can be traced back to the fact that $M_{1}$ behaves, for asymptotically large $M_{N_{2}}$ in eq.(3.15), as

$$
M_{1} \sim M_{N_{2}}+\frac{1}{M_{N_{2}}}\left[M_{N_{i}}\left(M_{N_{i}}-M_{2}\right)-\mu^{2}-M_{Z}^{2}+\frac{\mu}{M_{N_{i}}}\left(\mu M_{2}-M_{W}^{2} \sin 2 \beta\right)\right]+O\left(\frac{1}{M_{N_{2}}^{2}}\right)
$$

where $M_{N_{i}}$ is any of the neutralino masses other than $M_{N_{2}}$. Strictly speaking, (4.1) is valid only if the $M_{N_{i}}$ 's become insensitive to the value of $M_{N_{2}}$ in this limit. That this is true can be easily seen from the fact that all the $a_{i}$ 's in Eq. (B.7) behave in this limit like $\sim M_{N_{2}}^{3}$. Then the dependence on $M_{N_{2}}$ tends to cancel out in Eq.(B.6), making the three neutralino masses insensitive to this parameter, as illustrated in fig. 3 .

\subsection{GUT scale values of the ino mass parameters}

Next, in fig. 1 we illustrate plots very similar to the ones in fig. 2, but where in addition the parameters $\mu, M_{2}, M_{1}$ have been evolved from a low scale, $Q_{l o w} \sim 200 \mathrm{GeV}$, to a GUT scale, $Q_{G U T} \sim 2 \times 10^{17} \mathrm{GeV}$ according to the renormalization group evolution (RGE) [12]. In order to illustrate as simply as possible some representative values after RG evolution, note that we have made a number of approximations for the evolution itself, that we feel are reasonable for the present purpose. Namely, the RG evolution is limited to the one-loop approximation, and we have assumed in addition a single, universal supersymmetric threshold, that is identified with the low energy scale, $Q_{\text {low }} \sim 200 \mathrm{GeV}$, where the evolution starts (so that the RG evolution is dictated by fully supersymmetric beta function coefficients between $Q_{l o w}$ and $Q_{G U T}$ ). There should be no difficulties in principle to incorporate in our framework more realistic supersymmetric threshold effects, provided however that the masses of other partners (Higgses and sfermions) are known at this stage in addition to ino masses. The latter refinements should however produce relatively small 
corrections to the numerical values that are illustrated in fig. 4 .

It should not be very surprising that RGE scarcely change the shape of the various plots, with respect to those in fig. 2, apart from a systematic shift and a slight change in the slopes. This is a direct consequence of the generic form of the one-loop evolution equations for the gaugino masses and the $\mu$ parameter, namely

$$
\frac{d m}{d t} \sim m \times \sum_{i} \mathcal{G}_{i}^{2}
$$

where $\mathcal{G}_{i}$ refers to either gauge or Yukawa couplings and $t$ is the evolution parameter. Taking into account the evolution of the couplings themselves, one then expects the mass parameters to behave like

$$
m(t) \sim m_{0} \times \prod_{i} \mathcal{G}_{i}(t)^{a_{i}} / \mathcal{G}_{i}(0)^{a_{i}}
$$

where $a_{i}$ is some numerical power and $m_{0}$ the mass parameter at some initial scale. [The latter behaviour is exact, at the one-loop level, for the gauge couplings and also for the Yukawa couplings at least in the small $\tan \beta$ regime.] Thus the only effect of the running is a coupling dependent rescaling of the inversion results.

\subsection{Scenario $S_{2}$ : one chargino plus two neutralinos input}

Next we illustrate the (probably phenomenologically more relevant) scenario $S_{2}$, namely with $M_{\chi_{1}^{+}}, M_{N_{2}}$ and $M_{N_{3}}$ as input. As expected, fig. 5 reflects the more algebraically involved inversion from the combined algorithm $S_{2}$ (with unknown $M_{\chi_{2}^{+}}$), with the possible occurrence of several distinct solutions for $\left(\mu, M_{1}, M_{2}\right)$, as discussed previously. However, apart from the relatively untidy behaviour in some narrow zones, the domains of unique and twofold (or more) solutions are relatively well separated over a wide range of the $M_{N_{2}}$ values. Moreover, the behaviour of the plots for the particular input values in fig. 5 turns out to be quite generic. The shaded regions again corresponds to a zone where at least one of the output parameters $\left(\mu, M_{1}, M_{2}\right)$ becomes complex-valued. In fig. (5) we only show on purpose a range of values such that all masses are relatively light, while for larger $M_{N_{2}}$ the dependence of $\mu, M_{2}, M_{1}$ upon the latter becomes simpler, almost linear, in accordance with the behaviour in the previous figures for scenario $S_{1}$ alone. There are also specific values of the input masses such that one of the solutions for $\mu, M_{1}$, or $M_{2}$ is becoming very large, due to an explicit pole in the analytic inversion, as reflected e.g. in one of the $\mu$ and $M_{2}$ solutions, for $M_{N_{2}} \simeq 80 \mathrm{GeV}$ and $M_{N_{2}} \simeq 123 \mathrm{GeV}$, respectively.

Also, the dependence of scenario $S_{2}$ upon $\tan \beta$ variations is relatively mild. In contrast, varying $M_{\chi_{1}^{+}}$and/or $M_{N_{3}}$ input values can have more drastic effects, since in particular the occurrence of multi-fold solutions depends on those inputs.

Finally, for completeness, we show in fig. 6 the values of the other physical masses $M_{\chi_{2}^{+}}, M_{N_{1}}$ and $M_{N_{4}}$, resulting from the same input choice as in fig. 5. Note that, apart 
from $M_{N_{1}}$, the twofold valuedness of $M_{\chi_{2}}^{+}$and $M_{N_{4}}$ for $M_{N_{2}} \gtrsim 230 \mathrm{GeV}$ turns out to be numerically negligible for this particular input. In a more complete and realistic analysis, it should be possible to examine e.g. whether some of the multi-fold solutions in fig. 5 could be excluded by looking at the consistency of the resulting other physical ino masses with data.

\section{Other soft breaking parameters inversion}

Ultimately, one would need to reconstruct the remaining part of the soft-breaking Lagrangian, i.e. the sfermion and Higgs sectors. In contrast with the ino sector, the dediagonalization of these sectors would not present much analytical difficulties (at least at the tree-level), provided one knows a sufficient number of physical masses. A full strategy (including loop effects) is, however, beyond the scope of the present paper. Our aim here is to simply sketch for completeness some straightforward inversion formulas and make some comments.

\subsection{Sfermion parameter inversion}

In the sfermion sector, de-diagonalization is straightforward since it involves $2 \times 2$ mass matrices if neglecting flavor non-diagonal terms. For instance, from eqs. (A.6), and assuming the two physical stop masses $m_{\tilde{t}_{1}}, m_{\tilde{t}_{2}}$ (with the convention $m_{\tilde{t}_{1}}<m_{\tilde{t}_{2}}$ ) and the mixing angle $\theta_{\tilde{t}}$ to be given as input (plus $\tan \beta$ ), one immediately obtains

$$
A_{t}=\frac{\mu}{\tan \beta}+\left(m_{\tilde{t}_{2}}^{2}-m_{\tilde{t}_{1}}^{2}\right) \frac{\sin 2 \theta_{\tilde{t}}}{2 m_{t}}
$$

and

$$
\begin{gathered}
M_{Q}^{2}=m_{\tilde{t}_{1}}^{2} \cos ^{2} \theta_{\tilde{t}}+m_{\tilde{t}_{2}}^{2} \sin ^{2} \theta_{\tilde{t}}-m_{t}^{2}-\cos (2 \beta)\left(4 m_{W}^{2}-m_{Z}^{2}\right) / 6 \\
M_{R}^{2}=m_{\tilde{t}_{1}}^{2} \sin ^{2} \theta_{\tilde{t}}+m_{\tilde{t}_{2}}^{2} \cos ^{2} \theta_{\tilde{t}}-m_{t}^{2}+\frac{2}{3} \cos (2 \beta)\left(m_{W}^{2}-m_{Z}^{2}\right)
\end{gathered}
$$

Similar expressions are obtained for the sbottom and stau parameters.

Evidently, in the case of a more constrained scenario, one would not need all sfermion physical masses and mixing angles to extract the relevant soft terms. In fact, even in the unconstrained MSSM case, $M_{Q}$ is a common parameter to stop and sbottom mass matrices, so that to reconstruct the complete soft terms of this sector one "only" needs five parameters, to be chosen among the four masses and two mixing angles. Conversely, notice that if knowing the six previous physical input, one can determine $\tan \beta$ very simply from

$$
m_{W}^{2} \cos 2 \beta=m_{\tilde{t}_{1}}^{2} \cos ^{2} \theta_{\tilde{t}}+m_{\tilde{t}_{2}}^{2} \sin ^{2} \theta_{\tilde{t}}-m_{\tilde{b}_{1}}^{2} \cos ^{2} \theta_{\tilde{b}}-m_{\tilde{b}_{2}}^{2} \sin ^{2} \theta_{\tilde{b}}+m_{b}^{2}-m_{t}^{2}
$$




\subsection{Higgs sector inversion}

Going from physical masses to soft SUSY-breaking parameters in the Higgs sector also does not involve much difficulties, at least naively. In fact, electroweak symmetry breaking (EWSB) gives two (necessary but not sufficient) constraints, commonly used to fix the $B$ and $\mu$ parameter at the EWSB scale:

$$
\begin{array}{r}
\mu^{2}=\frac{m_{H_{d}}^{2}-\tan ^{2} \beta m_{H_{u}}^{2}}{\tan ^{2} \beta-1}-\frac{m_{Z}^{2}}{2} ; \\
B \mu=\left(m_{H_{d}}^{2}+m_{H_{u}}^{2}+2 \mu^{2}\right) \sin \beta \cos \beta .
\end{array}
$$

Now, one may assume for example that $\mu$ is extracted from the previous ino inversion, and use eqs. (5.4), together with the definition of the physical Higgs masses, eqs. (A.5), to determine the basic Higgs parameters. Typically, given the lightest CP-even higgs mass $M_{h}$, one obtains for the soft parameters at the tree-level:

$$
\begin{gathered}
m_{H_{u}}^{2}=\frac{M_{A}^{2}-\left(\mu^{2}+m_{Z}^{2} / 2\right)\left(\tan ^{2} \beta-1\right)}{1+\tan ^{2} \beta} ; m_{H_{d}}^{2}=M_{A}^{2}-M_{H_{u}}^{2}-2 \mu^{2} \\
B=\left(m_{H_{d}}^{2}+m_{H_{u}}^{2}+2 \mu^{2}\right) \frac{\sin (2 \beta)}{2 \mu}
\end{gathered}
$$

and

$$
M_{A}^{2}=\frac{M_{h}^{2}\left(m_{Z}^{2}-M_{h}^{2}\right)}{m_{Z}^{2} \cos ^{2} 2 \beta-M_{h}^{2}}
$$

and the other physical Higgs masses are determined from eqs. (A.5). Of course as is well known radiative corrections to the Higgs masses (in particular that of the lightest CP-even) are very important to take into account [13, 15], for some values of the sfermion masses and mixing angles, so that it is much too unrealistic to restrict to such a tree-level Higgs parameter inversion. Including the leading one-loop corrections in expressions 5.5 - 5.7) is in fact manageable. In this case the induced dependence on the full parameter set of the MSSM should be in principle taken into account. In particular, the parameters of the Higgs sector we are solving for enter now in a more complicated way equations (5.4). However, a very good starting point would be to consider only the dominant top/stopbottom/sbottom contributions in which case the corrections to Eqs.(5.5 - 5.7) can still be written in an analytical form. Otherwise, one will still have to resort to a thoroughly numerical procedure.

\section{Discussion and outlook}

In this paper we have worked out a systematic inversion algorithm and strategy to obtain from relatively simple algebraic relations the basic MSSM Lagrangian, from some appropriate physical mass inputs. We mostly concentrated on the ino parameter inversion, which is the less straightforward algebraically, due to a complicated diagonalization 
structure. The advantage over possibly more direct methods (like e.g. some systematic scanning of the Lagrangian parameter space), is that it gives a fast, reasonably tractable algorithm, and also clearly points out to the existence of non-trivial ambiguities in such a reconstruction, occurring in some definite range of the input mass values 9 .

In addition, our results exhibit the relatively simple and generic behaviour of most of the Lagrangian parameters as functions of input masses, apart from some narrow regions where the behaviour is more involved. If developed further, performing more systematic simulations, this approach may thus potentially give useful insight into the precise connection between the basic MSSM parameters and the experimentally measured ones. In the most optimistic case (that is, if knowing a sufficient number of physical input ino masses and with sufficient accuracy) it allows for a precise reconstruction of the unconstrained MSSM ino sector Lagrangian. In addition, some of the theoretically well-motivated additional assumptions, like for instance the universality of soft mass parameters, should be easily implementable in our basic algorithm, due to its relative flexibility. Typically, gaugino mass parameter universality at the GUT scale, leading to the approximate relation $M_{1}\left(M_{Z}\right) \simeq 5 / 3 \tan ^{2} \theta_{W} M_{2}\left(M_{Z}\right)$, can be very simply combined with our iterative algorithm $S_{2}$, to give now $\mu, M_{2}$ and $M_{1}$ in terms of only one chargino and one neutralino mass.

Finally we also mention the complementarity of our approach with the one discussed recently in ref. [10]. In particular, our simple analytical determination of $M_{1}$, eq. (3.15), may be readily implemented as well in their construction. Both procedures are however purely theoretical, in the sense that the influence of experimental errors on the input parameter measurements have not yet been taken into account. In any case, clearly an algebraic approach is a useful tool for a more systematic phenomenological study (taking into account e.g. the present bounds or the expected future accuracy on input parameters) to be presented elsewhere [16].

\section{Acknowledgments:}

We thank Abdelhak Djouadi for useful discussions. This work is partially supported by the French GDR-Supersymétrie.

\section{A MSSM mass formulas}

We collect here for completeness different useful expressions for the sfermion, ino and Higgs mass matrices or eigenmasses.

-Gaugino sector:

the chargino mass matrix reads from Eqs. (2.4, 2.5)

$$
\mathcal{M}_{C}=\left(\begin{array}{cc}
M_{2} & \sqrt{2} m_{W} \sin \beta \\
\sqrt{2} m_{W} \cos \beta & \mu
\end{array}\right)
$$

\footnotetext{
${ }^{6}$ Our main algorithm will be soon available as a computer fortran code, on request to the authors.
} 
with the chargino mass eigenvalues:

$M_{\chi_{1,2}}^{2}=\frac{1}{2}\left[M_{2}^{2}+\mu^{2}+2 m_{W}^{2} \pm\left[\left(M_{2}^{2}-\mu^{2}\right)^{2}+4 m_{W}^{4} \cos ^{2} 2 \beta+4 m_{W}^{2}\left(M_{2}^{2}+\mu^{2}+2 M_{2} \mu \sin 2 \beta\right)\right]^{1 / 2}\right] ;$

and the diagonalization of the non-symmetric matrix (A.1) involves two mixing angles, $\phi_{ \pm}:$

$$
\begin{aligned}
& \tan 2 \phi_{-}=2 \sqrt{2} m_{W} \frac{\mu \sin \beta+M_{2} \cos \beta}{M_{2}^{2}-\mu^{2}-2 m_{W}^{2} \cos 2 \beta} \\
& \tan 2 \phi_{+}=2 \sqrt{2} m_{W} \frac{\mu \cos \beta+M_{2} \sin \beta}{M_{2}^{2}-\mu^{2}+2 m_{W}^{2} \cos 2 \beta}
\end{aligned}
$$

-Higgs sector:

the mass eigenvalues of the neutral CP-odd A, CP-even $h, H$ and charged $H^{+}$scalars read:

$$
\begin{aligned}
M_{A}^{2} & =m_{H_{u}}^{2}+m_{H_{d}}^{2}+2 \mu^{2}+\Delta_{A} ; \\
M_{H \pm}^{2} & =M_{A}^{2}+m_{W}^{2}+\Delta_{H+} \\
M_{h, H}^{2} & =\frac{1}{2}\left[M_{A}^{2}+m_{Z}^{2} \mp\left[\left(M_{A}^{2}+m_{Z}^{2}\right)^{2}-4 M_{A}^{2} m_{Z}^{2} \cos ^{2}(2 \beta)\right]^{1 / 2}\right]+\Delta_{h, H}
\end{aligned}
$$

where $\Delta_{A}, \Delta_{H+}$, and $\Delta_{h, H}$ denote radiative correction contributions, whose complicated expressions in one-loop approximations are given e.g. in refs. [15, 17].

-Sfermion sector:

The mass matrices read

$$
\begin{aligned}
\mathcal{M}_{\tilde{t}}^{2} & =\left(\begin{array}{cc}
M_{Q}^{2}+m_{t}^{2}+\left(\frac{2}{3} m_{W}^{2}-\frac{1}{6} m_{Z}^{2}\right) \cos 2 \beta & m_{t}\left(A_{t}-\mu / \tan \beta\right) \\
m_{t}\left(A_{t}-\mu / \tan \beta\right) & m_{t_{R}}^{2}+m_{t}^{2}-\frac{2}{3}\left(m_{W}^{2}-m_{Z}^{2}\right) \cos 2 \beta
\end{array}\right) \\
\mathcal{M}_{\tilde{b}}^{2} & =\left(\begin{array}{cc}
M_{Q}^{2}+m_{b}^{2}-\left(\frac{1}{3} m_{W}^{2}+\frac{1}{6} m_{Z}^{2}\right) \cos 2 \beta & m_{b}\left(A_{b}-\mu \tan \beta\right) \\
m_{b}\left(A_{b}-\mu \tan \beta\right) & m_{b_{R}}^{2}+m_{b}^{2}+\frac{1}{3}\left(m_{W}^{2}-m_{Z}^{2}\right) \cos 2 \beta
\end{array}\right)
\end{aligned}
$$

which after diagonalization give the stop and sbottom mass eigenvalues $m_{\tilde{t}_{1}}, m_{\tilde{t}_{2}} ; m_{\tilde{b}_{1}}$, $m_{\tilde{b}_{2}}$ respectively, and their mixing angles $\theta_{\tilde{t}}, \theta_{\tilde{b}}$.

The $\tilde{\tau}$ mass matrix has a similar structure as the $\tilde{b}$ matrix, eq. (A.7), with the replacements $M_{Q} \rightarrow M_{L}, m_{\tilde{b}_{R}} \rightarrow m_{\tilde{\tau}_{R}}, m_{b} \rightarrow m_{\tau}$, and appropriate D-terms.

\section{B De-diagonalization of $M$}

The following discussion can be carried out in terms of any pair of $\tilde{M}_{N_{i}}$ (see section 3.2 for notations). We choose for definiteness $\tilde{M}_{N_{1}}, \tilde{M}_{N_{4}}$ and make the convenient relabeling 
$\tilde{M}_{N_{1}} \equiv X, \tilde{M}_{N_{4}} \equiv Y$ and $Z=X Y$. At this level we aim at the determination of some general consistency constraints and the distinction between input or output parameters is only for convenience.

Using Eq.(3.14) to substitute for $Z$ in Eqs.(3.11 - 3.13), one obtains a set of three linear equations in, say, the variables $X, Y, M_{1}$. This system would give easily $X, Y$ and $M_{1}$ in terms of the other parameters in the form

$$
X=\frac{\Delta_{X}}{\Delta}, Y=\frac{\Delta_{Y}}{\Delta}, M_{1}=\frac{\Delta_{M_{1}}}{\Delta}
$$

where it not for the fact that generically $\Delta=0$ while $\Delta_{X} \neq 0$ and $\Delta_{Y} \neq 0$. Actually the same happens independently for the two subsystems Eqs.(3.11, 3.12) or Eqs.(3.11, 3.13) when one tries to solve for $X, Y$, leading respectively to

$$
X=\frac{\delta_{1}}{\delta} \sim Y
$$

and

$$
X=\frac{\delta_{2}}{\delta} \sim Y
$$

where $\delta=0$ and $\delta_{1}, \delta_{2}$ are generically non vanishing. The necessary and sufficient conditions for the existence of solutions are thus $\delta_{1}=0$ and $\delta_{2}=0$. They can be cast in the following form:

$$
\begin{aligned}
& P_{i j}^{2}+\left(\mu^{2}+M_{Z}^{2}-M_{1} M_{2}+\left(M_{1}+M_{2}\right) S_{i j}-S_{i j}^{2}\right) P_{i j} \\
& +\mu M_{Z}^{2}\left(c_{w}^{2} M_{1}+s_{w}^{2} M_{2}\right) \sin 2 \beta-\mu^{2} M_{1} M_{2}=0
\end{aligned}
$$

and

$$
\begin{aligned}
& \left(M_{1}+M_{2}-S_{i j}\right) P_{i j}^{2}+\left(\mu^{2}\left(M_{1}+M_{2}\right)+M_{Z}^{2}\left(c_{w}^{2} M_{1}+s_{w}^{2} M_{2}-\mu \sin 2 \beta\right)\right) P_{i j} \\
& +\mu\left(M_{Z}^{2}\left(c_{w}^{2} M_{1}+s_{w}^{2} M_{2}\right) \sin 2 \beta-\mu M_{1} M_{2}\right) S_{i j}=0
\end{aligned}
$$

where $S_{i j}=\tilde{M}_{N_{i}}+\tilde{M}_{N_{j}}$ and $P_{i j}=\tilde{M}_{N_{i}} \tilde{M}_{N_{j}}, i=1, \ldots, 4, j=1, \ldots, 4$ with $i \neq j$. Note that we used here the fact that equations (B.4) and (B.5) should hold for any pair of eigenvalues since we could have chosen as $(X, Y)$ any set $\left(\tilde{M}_{N_{i}}, \tilde{M}_{N_{j}}\right)$ with $i \neq j$.

When Eq.(B.4) is satisfied, Eqs.(3.11) and (3.12) become equivalent to each other, so that the system made of (3.11), (3.12) and (3.14) is solvable in terms of $X$ and $Y$. Similarly, Eq.(B.5) does the same for the system (3.11) (3.13) and (3.14). The bottom line here is that we have traded Eqs.(3.11 - 3.14) for the system (B.4), (B.5) which has, 
however, some welcome features suitable for our purpose. An immediate consequence is that, [within the strategy defined in section 3 where $M_{2}$ and $\mu$ are determined from the physical chargino masses $M_{\chi_{1}^{+}}, M_{\chi_{2}^{+}}$and $\left.\tan \beta\right]$, one can determine uniquely $M_{1}$ and any three neutralinos mass parameters, say $\tilde{M}_{N_{1}}, \tilde{M}_{N_{3}}, \tilde{M}_{N_{4}}$ once the fourth, say $\tilde{M}_{N_{2}}$ is given. Indeed, substituting $M_{1}$ from Eq.(B.4) into Eq.(B.5) one obtains the following cubic equation in $\tilde{M}_{N_{i}}, i \neq 2$,

$$
a_{1} \tilde{M}_{N_{i}}^{3}+a_{2} \tilde{M}_{N_{i}}^{2}+a_{3} \tilde{M}_{N_{i}}+a_{4}=0
$$

where

$$
\begin{aligned}
a_{1}= & \tilde{M}_{N_{2}}^{3}+M_{2}\left(\mu^{2}-\tilde{M}_{N_{2}}^{2}\right)-\tilde{M}_{N_{2}}\left(\mu^{2}+c_{w}^{2} M_{Z}^{2}\right)-c_{w}^{2} \mu M_{Z}^{2} \sin 2 \beta \\
a_{2}= & s_{w}^{2} M_{Z}^{2}\left(\tilde{M}_{N_{2}}-M_{2}\right)\left(\tilde{M}_{N_{2}}+\mu \sin 2 \beta\right)-M_{2} a_{1} \\
a_{3}= & s_{w}^{2} M_{Z}^{2}\left(\tilde{M}_{N_{2}}^{3}+\left(M_{2}-\tilde{M}_{N_{2}}\right)^{2} \mu \sin 2 \beta+M_{2} \tilde{M}_{N_{2}}\left(M_{2}-2 \tilde{M}_{N_{2}}\right)\right)-\left(\mu^{2}+M_{Z}^{2}\right) a_{1} \\
a_{4}= & \mu\left[s_{w}^{2} M_{Z}^{2}\left(M_{2}\left(M_{2}-\tilde{M}_{N_{2}}\right)\left(\mu+\tilde{M}_{N_{2}} \sin 2 \beta\right)+c_{w}^{2} M_{Z}^{2} \sin 2 \beta\left(\mu \sin 2 \beta+\tilde{M}_{N_{2}}\right)\right)\right. \\
& \left.\quad+\left(\mu M_{2}-c_{w}^{2} M_{Z}^{2} \sin 2 \beta\right) a_{1}\right]
\end{aligned}
$$

The three solutions of Eq.(B.6) are given by

$$
\begin{gathered}
\tilde{M}_{N_{1}}=\frac{1}{3 a_{1}}\left(-a_{2}-\frac{\sqrt[3]{2} A}{B}+\frac{B}{\sqrt[3]{2}}\right) \\
\tilde{M}_{N_{3}}=\frac{1}{3 a_{1}}\left(-a_{2}+\frac{(1+I \sqrt{3}) A}{\sqrt[3]{4} B}-\frac{(1-I \sqrt{3}) B}{2 \sqrt[3]{2}}\right) \\
\tilde{M}_{N_{4}}=\frac{1}{3 a_{1}}\left(-a_{2}+\frac{(1-I \sqrt{3}) A}{\sqrt[3]{4} B}-\frac{(1+I \sqrt{3}) B}{2 \sqrt[3]{2}}\right)
\end{gathered}
$$

with

$$
\begin{gathered}
B=\sqrt[3]{C+\sqrt{4 A^{3}+C^{2}}} \\
A=-a_{2}^{2}+3 a_{1} a_{3} \\
C=-2 a_{2}^{3}+9 a_{1} a_{2} a_{3}-27 a_{1}^{2} a_{4}
\end{gathered}
$$

These solutions correspond necessarily to the three unknown neutralino mass parameters $\tilde{M}_{N_{1}}, \tilde{M}_{N_{3}}, \tilde{M}_{N_{4}}$, due to the fact that, as we mentioned before, Eqs. (B.4, B.5) should be satisfied for any pair $\left(\tilde{M}_{N_{i}}, \tilde{M}_{N_{j}}\right)$ with $i \neq j$. Furthermore, injecting any of these solutions back into Eq.(B.4) determines the same and unique value for $M_{1}$ given in Eq.(3.15). Finally one can cast Eqs.(B.8- B.10) in an even simpler form once it is realized that 
$\tilde{M}_{N_{1}}, \tilde{M}_{N_{3}}, \tilde{M}_{N_{4}}$ and $M_{1}$ are automatically real-valued when $M_{2}, \mu$ (and $\tilde{M}_{N_{2}}$ ) are taken real.

Indeed, when $\tilde{M}_{N_{2}}, M_{2}$ and $\mu$ are all real valued, i.e. $a_{1}, a_{2}, a_{3}$ and $a_{4}$ real, the cubic equation (B.6) assumes at least one real solution. The real-valuedness of $M_{1}$ follows then immediately from Eq.(3.15). However, since the latter equation should be satisfied for any pair $\left(\tilde{M}_{N_{i}}, \tilde{M}_{N_{j}}\right)$ with $i \neq j$, then all the remaining neutralino mass eigenvalues must also be real.

It thus follows that one can rewrite Eqs.(B.8- B.10) in an explicitly real valued form. To do this, we note first that the simultaneous reality of the $\tilde{M}_{N_{i}}$ 's is equivalent to

$$
1+\sqrt[3]{4} \frac{A}{|B|^{2}}=0
$$

implying that $A$ is necessarily a negative real-valued quantity. Plugging the above relation back into Eqs.(B.8 - B.10) one finds Eqs.(3.16 - 3.18) 


\section{References}

[1] For reviews see: H.P. Nilles, Phys. Rep. 110, 1 (1984);

H.E. Haber and G.L. Kane, Phys. Rep. 117, 75 (1985);

[2] see for instance S. P. Martin, hep-ph/9709356, to appear in Perspectives in Supersymmetry, ed. by G. L. Kane, World Scientific;

[3] L. Girardello and M.T. Grisaru, Nucl.Phys. B194 (1982) 65;

[4] A. H. Chamseddine, R. Arnowitt and P. Nath, Phys.Rev.Lett. 49 (1982) 970; R. Barbieri, S. Ferrara and C. A. Savoy, Phys.Lett. B119 (1982) 343; L. Hall, J. Lykken and S. Weinberg, Phys.Rev. D27 (1983) 2359;

[5] M. Dine and A. E. Nelson, Phys.Rev.D48 (1993) 1277;

[6] For a review on the Higgs sector of the MSSM, see J.F. Gunion, H.E. Haber, G.L. Kane and S. Dawson, "The Higgs Hunter's Guide", Addison-Wesley, Reading 1990.

[7] I. Hinchliffe et al., Phys. Rev. D55 (1997) 5520; CMS Collaboration (S. Abdullin et al.), CMS-NOTE-1998-006, hep-ph/9806366;

[8] D. Denegri, W. Majerotto and L. Rurua, CMS NOTE 1997/094, HEPHY PUB 678/97, hep-ph/9711357;

[9] M. El Kheishen, A. Shafik and A Aboshousha, Phys.Rev. D45 (1992) 4345;

[10] See for instance S.Y. Choi et al, "Chargino Pair Production in $e^{+} e^{-}$Collisions, hepph/9806279;

[11] S. Coleman, E. Weinberg, Phys. Rev. D 7 (1973) 1888;

[12] K. Inoue et al. Prog. Theor. Phys 68, (1982) 927; L. Ibañez and G.G. Ross Phys. Lett. B110 (1982) 227; L. Alvarez-Gaumé, J.Plochinski and M.B. Wise, Nucl. Phys. B250 (1983) 495; J. Ellis et al. Phys. Lett. B125 (1983) 2275; L.Ibañez and C. Lopez, Phys.Lett. 126B (1983) 54; Nucl.Phys. B233 (1984) 511; N.K. Falck, Z. für Phys. C30 (1986) 247; see also for instance R. Arnowitt and P. Nath, Lecture at Swieca School, Campos do Jordao, Brazil, Jan. 1993, and references therein;

[13] J.Ellis, G. Ridolfi, F. Zwirner, Phys. Lett. B257 (1991) 83; H.E. Haber and R. Hempfling, Phys.Rev.Lett. 66 (1991) 1815; R. Barbieri, M. Frigeni and M. Caravaglios Phys. Lett. B258 (1991) 167; Y. Okada, M. Yamaguchi and T. Yanagida, Prog. Theor. Phys. 85 (1991) 1;

[14] see for instance, D.J. Castaño, E.J. Piard and P. Ramond, Phys.Rev.D49 (1994) 4882; W. de Boer, R. Ehret and D.I. Kazakov, Z. Phys. C67 (1994) 647; V. Barger, M.S. Berger and P. Ohmann, Phys.Rev.D49 (1994) 4908; 
[15] M. Carena, M. Quiros and C.E.M. Wagner, Phys. Lett. B335 (1995) 209.

[16] A. Djouadi, J.L Kneur and G. Moultaka, in preparation.

[17] For a review see for instance H.E. Haber, "Higgs Boson Masses and Couplings in the Minimal Supersymmetric Model" and references therein, (hep-ph/9707213) to appear in Perspectives on Higgs Physics II Gordon L. Kane editor; 


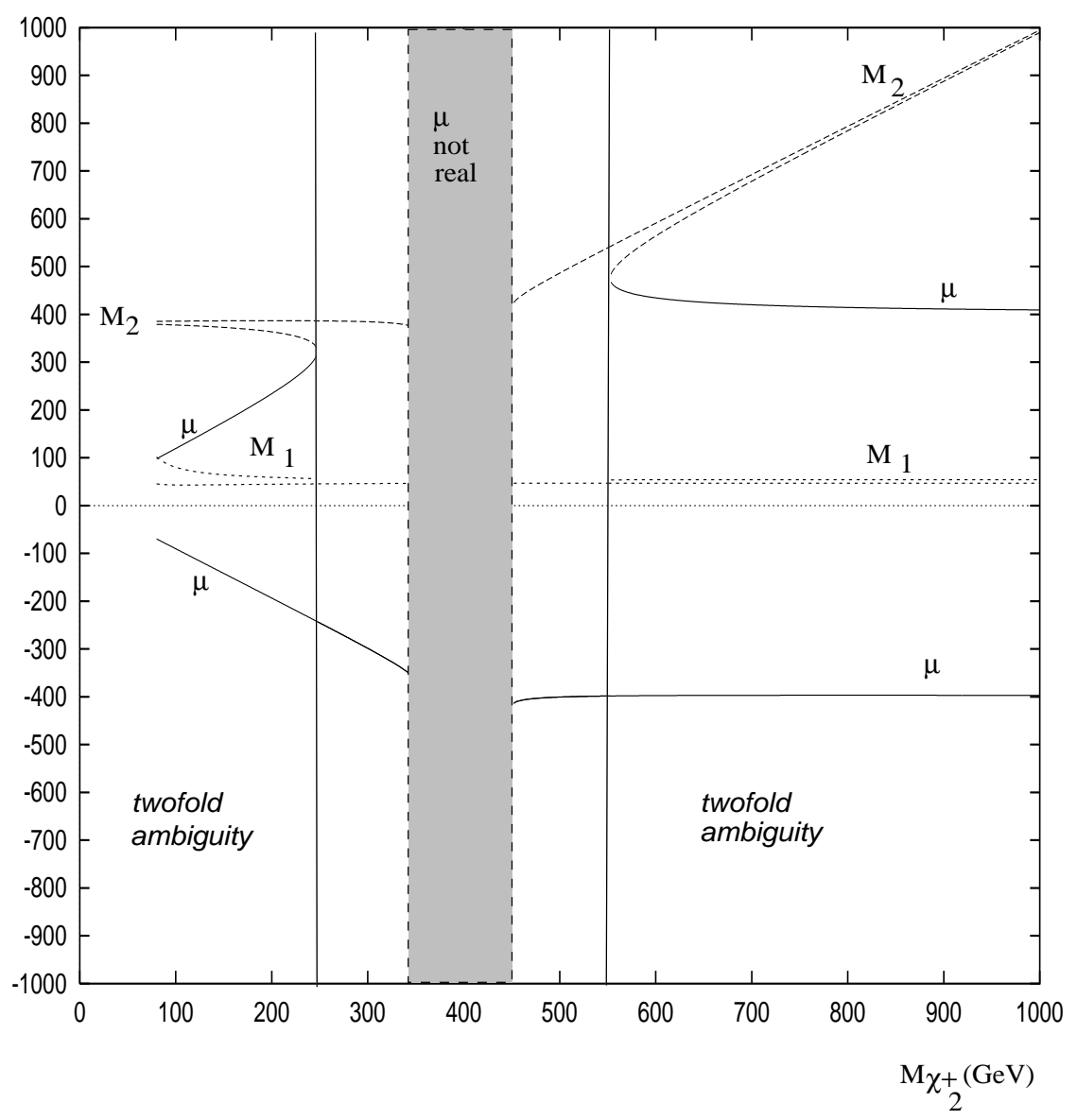

Figure 2: $\quad \mu, M_{2}$ and $M_{1}$ (with the "higgsino-like" convention $|\mu| \leq M_{2}$ ) as functions of $M_{\chi_{2}^{+}}$for fixed $M_{\chi_{1}^{+}}(=400 \mathrm{GeV}) ; M_{N_{2}}(=50 \mathrm{GeV})$, and $\tan \beta(=2)$. The opposite "gaugino-like" situation, with $|\mu| \geq M_{2}$, is trivially obtained by the substitution $M_{2} \rightarrow|\mu|$ and $\mu \rightarrow \operatorname{sign}(\mu) M_{2}$. 


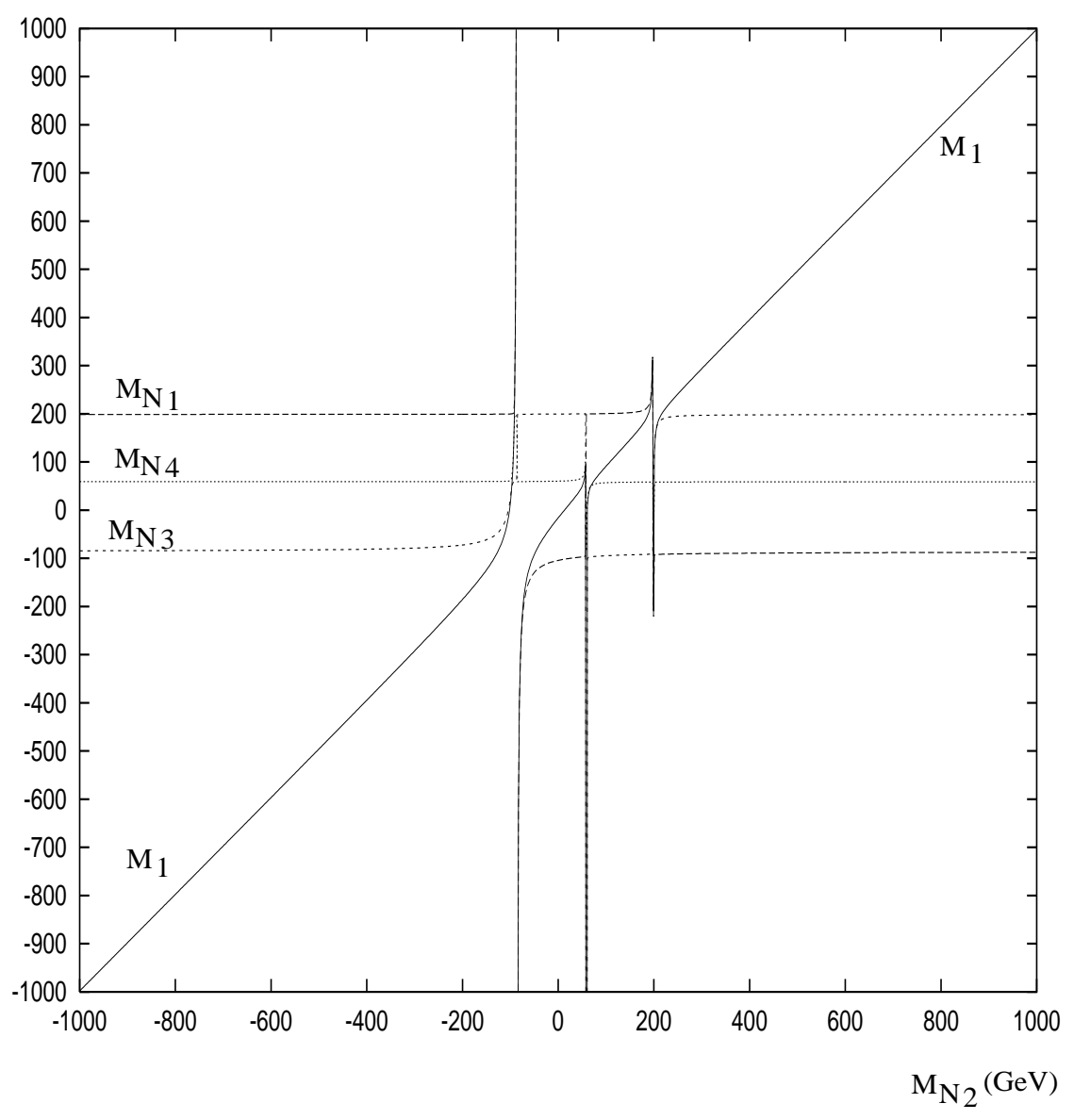

Figure 3: $\quad M_{1}$ and the three neutralino masses $\left(M_{N_{1}}, M_{N_{3}}, M_{N_{4}}\right)$ as functions of $M_{N_{2}}$ for fixed $M_{\chi_{1}^{+}}(=80 \mathrm{GeV}), M_{\chi_{2}^{+}}(=200 \mathrm{GeV})$ and $\tan \beta(=2)$. 


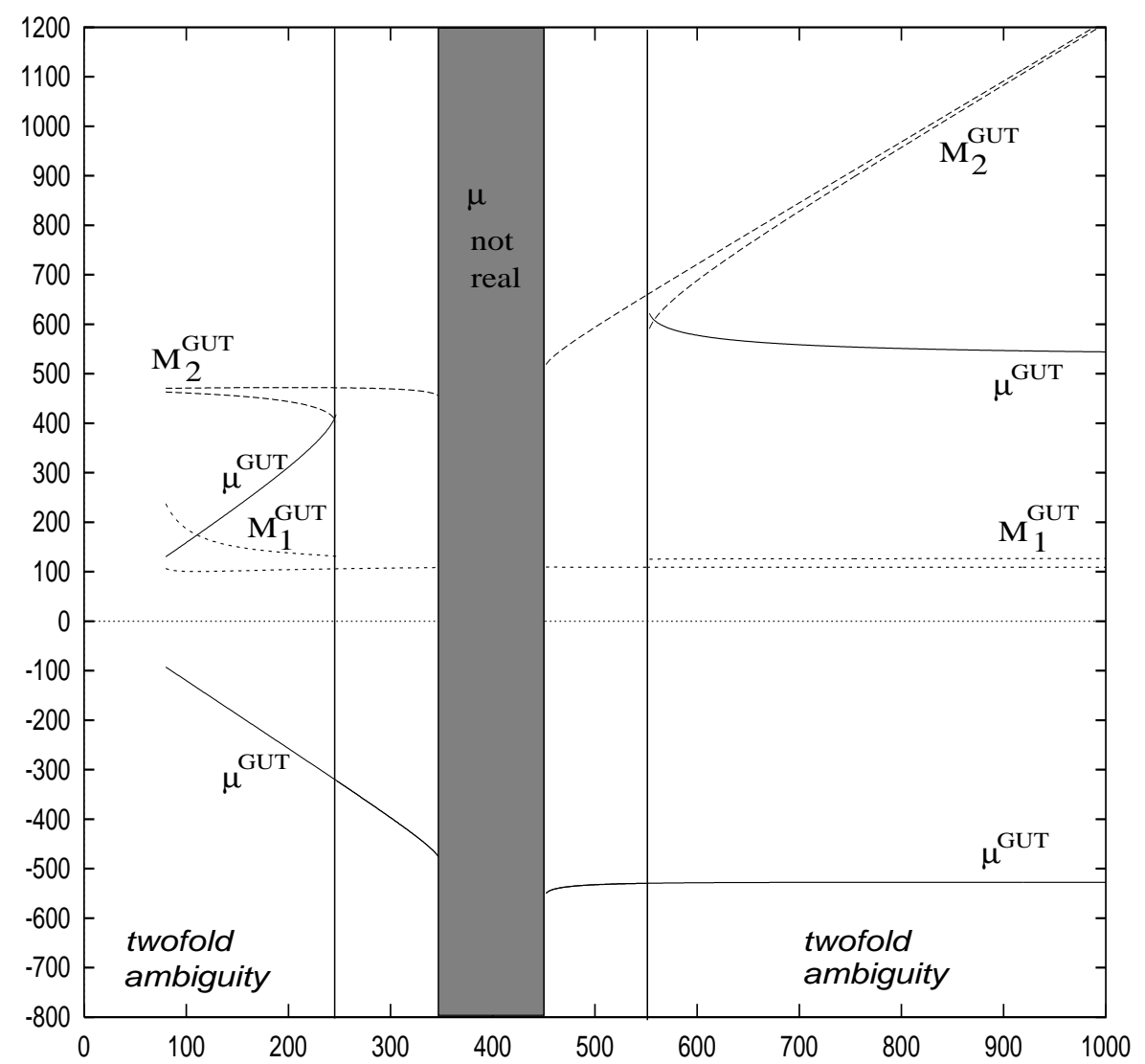

$\mathrm{M}_{\chi_{+}}(\mathrm{GeV})$

Figure 4: Same captions as for fig. 2 but with $\mu, M_{2}$ and $M_{1}$ evolved up to GUT scale. 


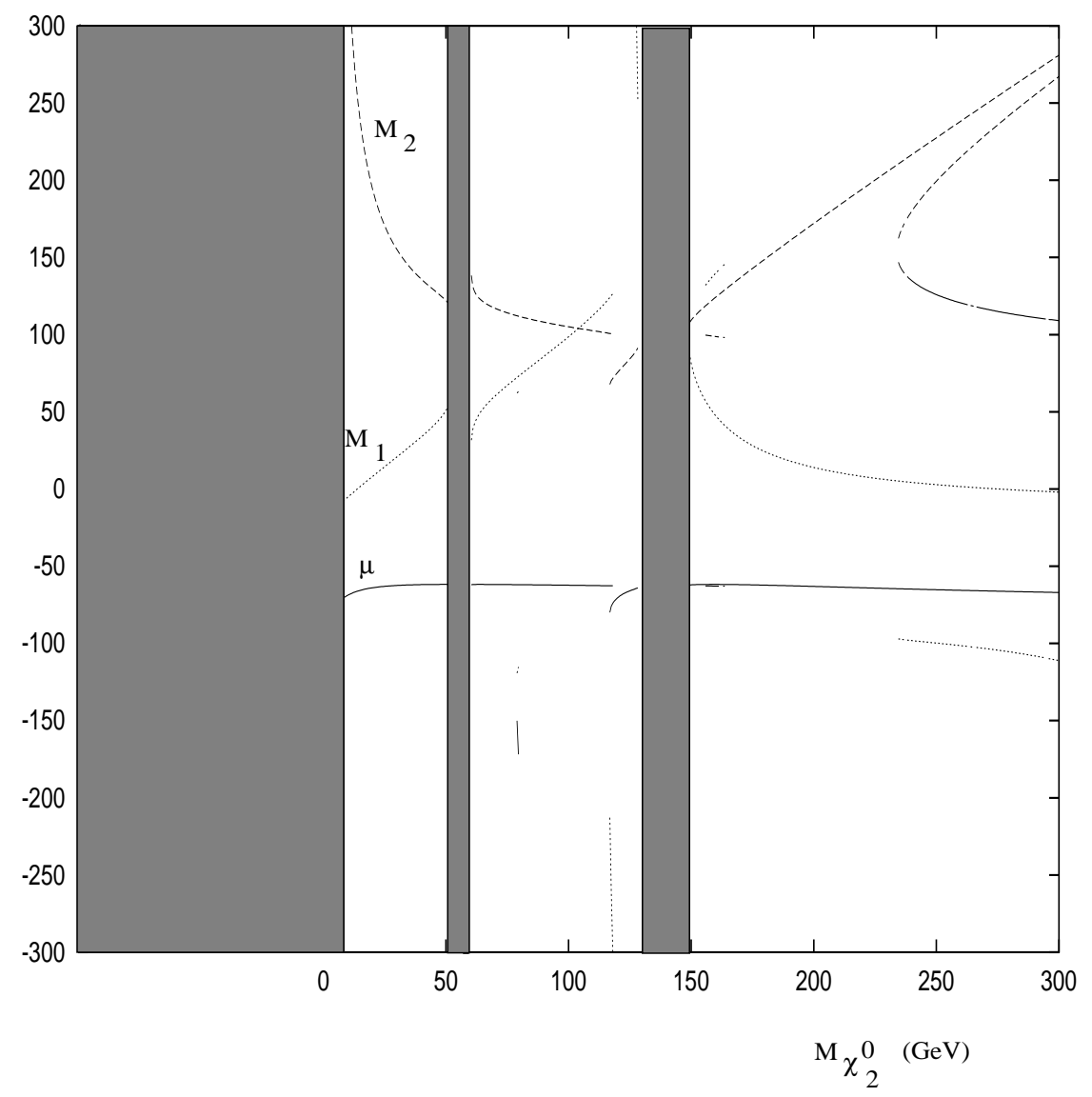

Figure 5: $\quad \mu, M_{2}$ and $M_{1}$ as function of $M_{\chi_{2}}^{0} \equiv M_{N_{2}}$ for fixed $M_{N_{3}}(=-100 \mathrm{GeV}), M_{\chi_{1}^{+}}$ $(=80 \mathrm{GeV})$ and $\tan \beta(=2)$. 


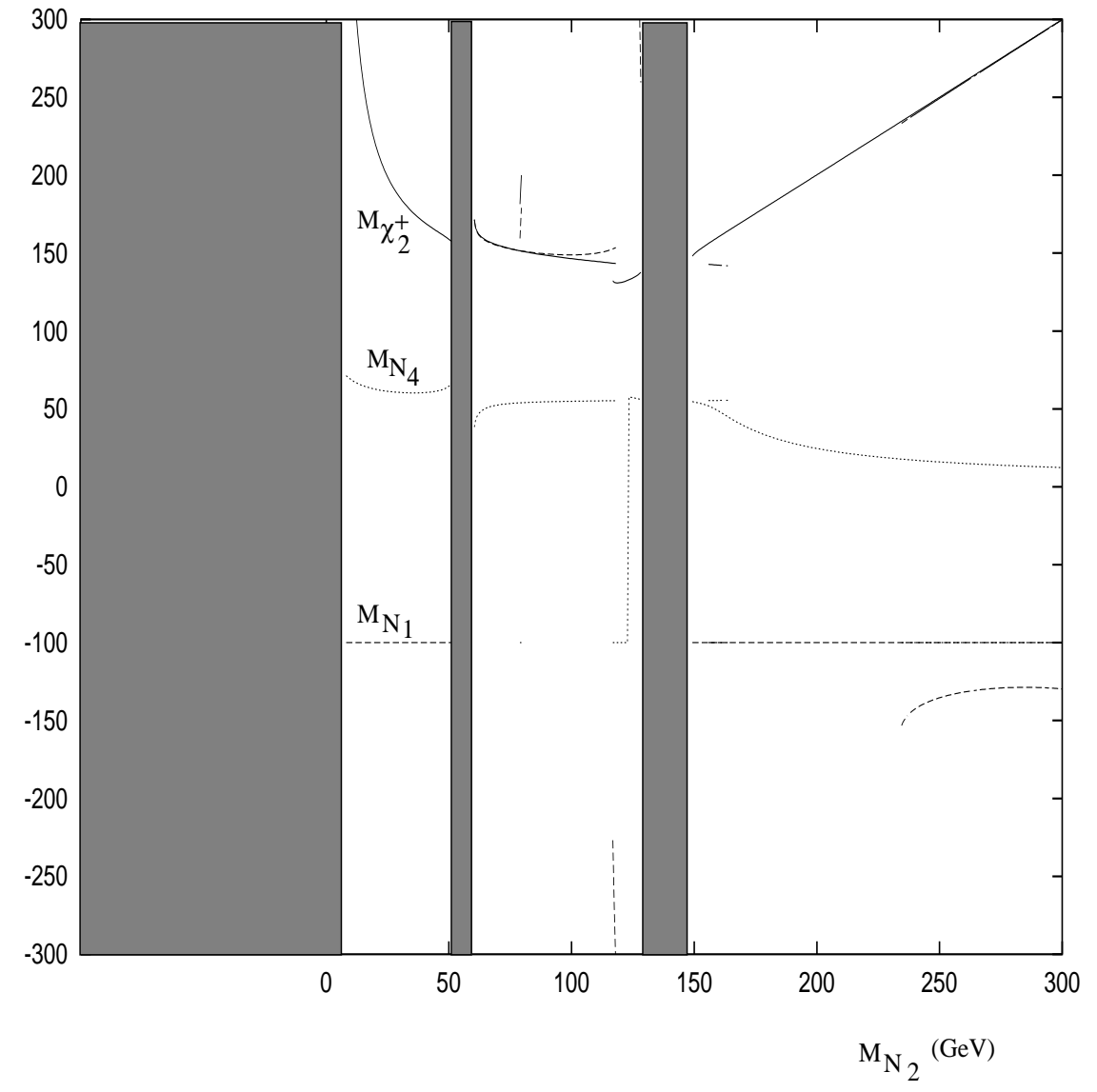

Figure 6: resulting $M_{\chi_{2}^{+}}, M_{N_{1}}$ and $M_{N_{4}}$ values for the $\mu, M_{2}, M_{1}$ values of fig. 5. 